\title{
Operational research for the introduction of an adolescent health package in the context of cervical cancer prevention
}

\author{
Marieke G. van Dijk \\ Population Council \\ Katherine Wilson \\ Population Council \\ Claudia Diaz \\ Sandra G. Garcia \\ Population Council
}

Follow this and additional works at: https://knowledgecommons.popcouncil.org/departments_sbsr-rh

Part of the Demography, Population, and Ecology Commons, Family, Life Course, and Society Commons, Gender and Sexuality Commons, International Public Health Commons, Medicine and Health Commons, and the Women's Health Commons

How does access to this work benefit you? Let us know!

\section{Recommended Citation}

van Dijk, Marieke G., Katherine Wilson, Claudia Diaz, and Sandra G. Garcia. 2009. "Operational research for the introduction of an adolescent health package in the context of cervical cancer prevention," Final meeting report. Mexico City: Population Council. 
Operational Research for the introduction of an adolescent health package in the context of cervical cancer prevention

WHO, PAHO, UNFPA Meeting of Potential Study Collaborators

Mexico City, Mexico

February 25-27, 2009

Marieke van Dijk, Population Council Mexico

Katherine Wilson, Population Council Mexico

Claudia Diaz Olavarrieta, National Institute of Public Health, Mexico

Sandra G. Garcia, Population Council Mexico 


\section{Meeting Purpose:}

To discuss an operational research study on adolescent health and cervical cancer prevention including HPV vaccines, to help support countries that have already made, or will soon make a policy decision to introduce the HPV vaccine.

\section{Background:}

The Global Immunization Vision and Strategy (GIVS) of WHO and UNICEF calls for reaching broader populations with vaccines by extending immunization programs to all age groups. Adolescents typically have little contact with health services, particularly for immunization programs. Since new HPV vaccines target adolescent girls, the introduction of HPV vaccines may provide an opportunity for adolescents to engage more with health services. This may create openings to offer more than vaccination, but a package of adolescent health services that includes information, sexual and reproductive health education, and adolescent health interventions. To better understand the policy and programmatic issues related to delivering HPV vaccines as part of comprehensive adolescent health services and for cervical cancer prevention, and as stated in the WHO publication " Preparing for the introduction of HPV vaccines: policy and programme guidance for countries" (http://www.who.int/reproductivehealth/publications/hpvvaccines/text.pdf) a study is proposed.

With the recent adoption of resolutions by the $48^{\text {th }}$ Directing Council of the Pan American Health Organization on adolescent health and on comprehensive cervical cancer prevention and control, the region of the Americas has been given a political mandate to improve adolescent health services and cervical cancer prevention. Thus, an operational research study to assess the feasibility, acceptability, cost and means for service delivery and monitoring adolescent health services, including cervical cancer prevention and HPV vaccines would help support the implementation of these two resolutions. And as there are several countries in the Americas initiating or considering the introduction of the HPV vaccine, the timing for such a study is appropriate. A meeting of potential study collaborators is proposed to discuss the proposal for an operational research study and to refine and adapt the study objectives and protocol.

\section{Meeting objectives:}

1. To share information on country programs for adolescent health, sexual and reproductive health, cervical cancer prevention and HPV vaccine introduction.

2. To present the proposed WHO adolescent health package, in relation to sexual and reproductive health and cervical cancer prevention; and to discuss how the package could be adapted and delivered, in relation to current adolescent health services in countries. 
3. To discuss the proposed operational research study to assess the feasibility, acceptability, cost and means for service delivery and monitoring of adolescent health services, including cervical cancer prevention and HPV vaccines.

4. To define criteria for participating countries and study sites, collaborating research centers and researchers.

5. To develop a work plan for the operational research study, for each participating country.

\section{Expected Outcomes of the Meeting:}

1. A work plan for adaptation and development of the operational research study that will commonly be applied in all participating countries.

2. A work plan to implement the operational research study, for each participating country.

3. A suggested principal investigator for each country, and designated country steering research committee.

\section{Discussing the project to meeting participants:}

\section{The new HPV vaccine: an opportunity to improve adolescent's health services}

Representatives from several organizations (PAHO, WHO, UNFPA-Mexico, Center on Equity and Reproductive Health, Ministry of Health Mexico - CERH- and the office on Prevention and Control of HIV-AIDS-Mexico) discussed the importance of this meeting that will allow participating countries the opportunity to share initiatives on the introduction of the HPV vaccine and the ways in which introducing the vaccine can improve health services for adolescents in four countries in the region of Latin America and the Caribbean (Colombia, Peru, Panamá y México). The meeting would give participating countries the opportunity to work as a group among different organizations and across several programs (immunization, prevention of cervical cancer, reproductive and adolescent health).

The seminal idea of using the HPV vaccine to offer health services to adolescents came from the finding that adolescents tend not to access health services. In places where there is access to specific packages for adolescent health, providers may not implement them for several reasons (e.g. they do not know these packages are available, they do not perceive adolescents as a group at risk, and they have concerns about the legal implications of needing informed consent from parents of minors before they offer any health service). The groups from participating countries in this meeting are either considering the introduction of the HPV vaccine at the national level or have already done so and aim to expand the package of services. 
The objective of this meeting was to discuss the feasibility of introducing the HPV vaccine in combination with a comprehensive package of adolescent health services and assess the strategy via a research protocol that follows an operations research (OR) methodology.

\section{The HPV vaccine as a key tool to prevent cervical cancer}

The current project is based on the scientific evidence gathered to date on the effectiveness of introducing the HPV vaccine to help prevent cervical cancer. This evidence and the programmatic guidelines to introduce the vaccine are described in the "green guide", a document drafted by WHO, UNFPA and PAHO entitled "Preparing for the introduction of HPV vaccines: policy and programme guidance for countries" (see Sharepoint, guide HPV-WHO).

HVP infection is the leading cause of cervical cancer. In most women, the infection disappears but chronic infection of some of the HPV genotypes can produce cellular changes in the cervical uterine region. Industrialized countries have been successful in reducing the death rates attributed to cervical cancer with integrated programs that combine: 1) information and education to women regarding the disease and ways to prevent it; 2) screening; 3) diagnosis and treatment; and 4) palliative care. Introducing the HPV vaccine could strengthen these programs by preventing the infection by the HPV virus. There are two vaccines currently available: Gardasil and Cervarix that offer protection against four HPV strains and are administered in three doses. The vaccine is more effective when it is administered prior to the initiation of sexual activity.

Introducing the HPV vaccine, unlike other vaccines, creates important barriers due to factors such as its high cost and access to the target age group. First, introducing the vaccine requires coordinating different programs and institutions including vaccination programs, cervical control programs, overall health system delivery and the Ministry of Education, among others. The decision to introduce a vaccine must be based on the burden of disease, its cost-effectiveness, sustainability and, if the country in question has the resources and infrastructure needed to introduce it. The groups from each participating countries will have to define the feasibility of introducing the vaccine in their context, the strategy they will need to supply the vaccine. Important considerations include vaccine introduction (via health education campaigns or through schools, defining the appropriate target population and, if it is feasible, carrying out a "catch-up" For adolescents who have not previously been linked into the health system. Legal aspects such as informed consent and whether the vaccine would be obligatory must be considered together with other factors such as the developmental stage of adolescents.

\section{Comprehensive package of adolescent health services}

WHO suggests we capitalize on three points in time during which the HPV vaccine is/could be administered in order to offer a comprehensive package of health services or 
a "plus" package for adolescents. WHO carried out an extensive literature review and designed a "menu" of choices that could be included in such a package (see Table 1 and Sharepoint documents "WHO ADO HPV Lit Review Report 20081114 FINAL" and "HPV Intervention Package for Adolescent Health")

The types of components that could be included in the package are: screening (e.g. anemia, vision), delivery of information (sexual and reproductive health, HPV, nutrition), service provision (counseling, vouchers, referrals to other health services) and delivery of supplies (e.g. Vitamin A, mosquito nets, vaccines such as hepatitis B and tetanus). The contents of the package may vary according to the needs of each country as well as the most appropriate target population to be included in a planned OR study.

Table 1. Menu of options for a comprehensive package of health services for adolescents

\begin{tabular}{|c|c|c|}
\hline $\begin{array}{l}\text { Category } \\
\text { Intervention }\end{array}$ & Definition & $\begin{array}{l}\text { Menu of complementary } \\
\text { interventions }\end{array}$ \\
\hline Screening & $\begin{array}{l}\text { Test or screening for } \\
\text { diseases or disabilities }\end{array}$ & $\begin{array}{l}\text { - Anemia screening } \\
\text { - Schistosomiasis screening } \\
\text { - } \text { Visual screening (in appropriate } \\
\text { contexts) }\end{array}$ \\
\hline $\begin{array}{l}\text { Information } \\
\text { provision }\end{array}$ & $\begin{array}{l}\text { Delivery of interventions } \\
\text { based on information, } \\
\text { included in information } \\
\text { sessions, skill building, } \\
\text { pamphlets. }\end{array}$ & $\begin{array}{l}\text { - Information on nutrition } \\
\text { - Information on Sexual and } \\
\text { Reproductive Health } \\
\text { - HPV information } \\
\text { - Information on tobacco and } \\
\text { alcohol prevention }\end{array}$ \\
\hline Services & $\begin{array}{l}\text { Referral to other health } \\
\text { services, counseling, } \\
\text { treatments }\end{array}$ & $\begin{array}{l}\text { - Referral to "vouchers" for health } \\
\text { services (including SRH). } \\
\text { - Referral to visual screening } \\
\text { - } \quad \text { Referral to delivery of } \\
\text { iron/folate supplements }\end{array}$ \\
\hline $\begin{array}{l}\text { Delivery of } \\
\text { supplies }\end{array}$ & $\begin{array}{l}\text { Supplies, direct provision } \\
\text { of basic products or } \\
\text { increased access to basic } \\
\text { products }\end{array}$ & $\begin{array}{l}\text { - Antihelmintics (transmitted by } \\
\text { contact with floor) } \\
\text { - Antihelmínticos } \\
\text { (schistosomiasis) } \\
\text { - Mosquito nets for prevention of } \\
\text { malaria } \\
\text { - Vitamin A }\end{array}$ \\
\hline Vaccines & $\begin{array}{l}\text { The vaccines identified to } \\
\text { complement the } \\
\text { administration of the HPV } \\
\text { vaccine (primary or }\end{array}$ & $\begin{array}{l}\text { Tetanus/ Diphteria booster dose } \\
\text { Hepatitis B ( booster) } \\
\text { Costa Rica ( booster ADO P) } \\
\text { Meningococal (only to specific groups }\end{array}$ \\
\hline
\end{tabular}




\begin{tabular}{|l|l|}
\hline booster) for this age group & $\begin{array}{l}\text { and high risk areas in LAC) } \\
\text { Typhoid (only for outbursts) } \\
\text { Rubeola (SR booster) }\end{array}$ \\
\hline
\end{tabular}

Introducing the HPV vaccine and the comprehensive package of adolescent health services in the region of Latin America and the Caribbean: a strategic opportunity

The LAC region has the largest cohort of young adults aged 10-24 and the most inequitable distribution of income in the world. Most of these young people come from low income strata and have a high prevalence of unwanted pregnancies. Implementing this project constitutes a strategic opportunity since the political climate seems ripe for it. In 2008, El Salvador hosted a regional meeting that convened presidents and key stakeholders who drafted a youth plan aimed at improving the sexual and reproductive health of adolescents for the next ten years. The HPV vaccine could constitute an important opportunity to address all forms of inequality in the region.

In May of the same year, 2008 a meeting was organized in Mexico to discuss the state of the art and recent developments in the field of the HPV vaccine ("Towards the prevention and comprehensive control of cervical cancer"- see Sharepoint, document HPV_MEX Declaration).

A few months later in September 2008, the organizing committee from PAHO and WHO drafted a resolution entitled "Strategy and regional action plan for the prevention and control of cervical cancer" (see Sharepoint, document CD48 R10 Cancer cervicouterino-Esp). During this meeting, the committee called the member states to introduce the HPV vaccine if the price was feasible and financing became sustainable. PAHO offered technical assistance to strengthen integrated cervical cancer programs and support decision makers who showed an interest in introducing the vaccine.

\section{Presentations on the current situations in participating countries with respect to adolescent health, the HPV vaccine, and opportunities for introducing a comprehensive adolescent health package}

Each country presented their country profile, the way their health care system was organized, current status of adolescent health and an update on the HPV vaccine. For more detailed information you can access the power point presentations in Sharepoint.

\section{Mexico - Dr. Patricia Uribe (National Center on Equity and RH-MOH)}

Context and adolescent needs: The Federal $\mathrm{MOH}$ has several programmatic areas that oversee adolescent health. The National Center on Equity and RH (NCERH) is in charge of the cervical cancer program and currently does not have the technical expertise or experience working with adolescent health. The most common health problems among adolescents in Mexico include: early initiation of sexual activity (20\% 
start between 15-19 years); pregnancy at an early age (18 years in rural areas); and a gap between knowledge and practice. For example, n spite of having high levels of knowledge of contraceptive methods, $90 \%$ report having had their first sexual experience without protection. Other problems include the increasing incidence of sexually transmitted infections and high rates of dating violence in this age group. The $\mathrm{MOH}$ has designed youth friendly services to avoid the barriers associated with traditional services such as lack of knowledge of bureaucratic procedures from the patient's perspective, lack of knowledge of legal aspects of service delivery to minors from the provider's perspective, office hours that are not convenient to adolescents, among others. As is common in most countries, women are more frequent users of health services than men and tend to seek care for issues regarding maternal health.

Cervical cancer context: In Mexico, cervical cancer is still the leading cause of cancerrelated death in the poorest areas of the south that border with Central America (breast cancer is the leading cause of death at the national level). In these areas, cytology coverage is low. The National Institute of Public Health of Mexico carried out a pilot study on strategies aimed at decreasing the prevalence of cervical cancer. Based on their initial findings and following their technical advice, in 2008, the NCERH selected the 125 communities ("municipios") with the lowest level of human development indexes in the country and began an HPV vaccine program for all adolescent girls between 9 and 12 years. Due to budgetary restrictions, they were unable to include a larger number of communities. It has been shown that these communities have the highest risk of acquiring cervical cancer. As part of the intervention, DNA testing for cervical cancer was also offered to women. In 2009, the MOH plans to administer the HPV vaccine and DNA testing to more communities throughout the country. The intervention will stress the need to carry out the Papanicolau test as well as increase coverage of DNA testing at the national level. Similarly, the MOH plans on strengthening the quality of diagnosis and treatment that is currently offered free of charge to all women in Mexico. The HPV vaccine is currently administered thru the school system and community centers via a health education marketing campaign. While some challenges remain, the vaccine has been very well accepted and key stakeholders have the political and social will to carry out this project successfully.

The HPV vaccine: In 2008, the MOH purchased the HPV vaccine for $\$ 436.47$ pesos per dose (current exchange rate is 13.5 pesos per \$1 USD). The national health security system later bought it at a lower price of $\$ 327.00$ pesos per dose and the Mexico City $\mathrm{MOH}$ was able to buy it at $\$ 21.00$ USD as greater competition among the pharmaceutical companies that manufacture the vaccine arose. At the beginning, the vaccine was administered in the following schedule of 0, 2 and 6 months. Researchers are currently trying to define the feasibility of administering it at 0 and 2 months (for 9 yr olds) and at 60 months (for $15 \mathrm{yr}$ olds). This schedule is based on one implemented successfully in Quebec, Canada. The period of time between the second and third dose will provide ample time to determine the need for administering the third dose. 


\section{Panama - Dr. Yadira Carrera (Ministry of Health)}

Context and adolescent needs: As in the rest of the LAC region, Panama has a high rate of adolescent pregnancy (18\%). The Ministry of Health and Social Development of Panama has health programs targeted for adolescent and recently launched a teen pregnancy prevention program in collaboration with UNFPA (2006-2008). The country also has a comprehensive adolescent health program. Nevertheless, this program has access barriers such as lack of space for teenagers seeking medical care and provider training needs so that they can offer care to adolescents accompanied by their parents. In addition, the country has to face the challenge of the opposition from the Catholic Church towards the use and distribution of contraceptive methods.

Context of cervical cancer: HPV infection is the fifth leading cause of morbidity in Panama. At the national level, cervical cancer is the second leading cause of death followed by breast cancer. Most cases are concentrated in rural areas. The country has a cervical cancer screening program which is especially difficult to implement in rural areas due to the distrust associated with Pap tests among the population. The country needs to reinforce existing training among providers, health education campaigns as well as train laboratory personnel and develop adequate technical infrastructure to carry out screening tests.

The HPV vaccine: In October 2008, Panama added the HPV vaccine to their comprehensive immunization program. The vaccine is offered free of charge to 10 year old girls (10 to 10.11 months) in three doses. The vaccine will be offered through the public health system and in MOH clinics. They have developed the service provision delivery system for the vaccine.

\section{Peru - Dr. Lourdes Palomino (Ministry of Health) and Dr. Mario Martínez (PAHO)}

Context and adolescent needs: In Peru, one fifth of the population is of adolescent age, living in cities, $60 \%$ are migrants from rural areas and $20 \%$ have low levels of schooling. Rural areas have the highest rates of adolescent pregnancies. Contraceptive coverage is low, STI transmission is high and the country has high rates of gender based violence, including sexual violence. The $\mathrm{MOH}$ is currently undergoing health care sector reform and moving toward access to universal health care. The current strategies aimed at adolescents include sexual and reproductive health together with mental health services that are offered throughout 700 youth friendly clinics where adolescent access services using a "carnet." As part of these youth friendly services, the MOH has also included social and personal skill building that allow adolescents to act as community leaders in a peer-based service delivery system. 
Context of cervical cancer and the HPV vaccine: Dr. Martínez stressed the benefits that vaccines have in the epidemiology of diseases. Peru has a high rate of cervical cancer, especially in rural areas with extreme poverty that are difficult to access such as the jungle region. The HPV vaccine has not been introduced in Peru yet although the MOH carried out a pilot study with an international NGO (PATH) in three sites (Piura, Ayacucho and Ucayali) with the aim of assessing a future implementation strategy that measures acceptability among the population and among providers and determines the resources that will be needed and if the HPV vaccine constitutes a health priority in Peru. Girls enrolled in school participated in the study once their parents had given informed consent. The vaccine was administered in three doses at 0, 2 and 6 months. Over 1,000 girls received the vaccine and large coverage was achieved. The cost per girl for three doses was $\$ 90$ USD; this price included total costs such as transporting the vaccine, provider's salaries, etc.).

\section{Colombia - Sonia Bedoya (Government Province of Medellin) and Marion Piñeros (National Cancer Institute)}

Context and adolescent needs: In Colombia, $13.7 \%$ of women between ages 15 and 19 report having their first sexual relationship before they reach 15 years; $80 \%$ of women in that same age group currently do not use a contraceptive and only $38.2 \%$ have ever used a contraceptive method. These figures illustrate an important unmet need for family planning methods in Colombia. The $\mathrm{MOH}$ is actively working to strengthen adolescent health programs and facilitate access to services and methods.

Context of cervical cancer: Cervical cancer is the leading cause of death in Colombia. A National Health Plan 2007-2010 was drafted and one of its goals is to reduce the mortality associated to cervical cancer. Early screening and detection in the country is acceptable (77\% in the last 3 years) but they still have to work on coordinating screening, detection and treatment. Colombia has considered carrying out a pilot study focusing on the areas with the highest cervical cancer prevalence among the most vulnerable groups. A prior study documented the most common HPV strains associated with cervical cancer (16 and 18) were found to be most prevalent. The most aggressive forms of HPV were found among women between ages 15 and 30 and a second peak was found among women between ages 40 and 50 (often associated with sex outside of marriage).

The HPV vaccine: The Colombian government supports the potential introduction of the HPV vaccine. The factors they are taking into account include: burden of disease; costeffectiveness of the vaccine; and, logistical and financial sustainability. Because of the current political conditions in the country, the government has a long list of unmet health needs. However, each province has its own $\mathrm{MOH}$ and the discretionary power to allocate resources according to need and to add health priorities to the National Health Plan. The following ethical considerations will be taken into account when designing the study methodology: will they select all girls or will they select particular 
communities and administer the vaccine to all girls? How will they address the social reaction and doubts surrounding the vaccine's efficacy? How will they address the controversy of the booster dose? How will they design future screening programs? How will they be able to increase parental consent for minors? How will they address continuity of the vaccine for future cohorts? Researchers attending the meeting agreed that an operations research methodology may help clarify some of these questions. Colombia is planning on packaging the HPV vaccine as a strategy to prevent cervical cancer and not as a vaccine to prevent STIs. The $\mathrm{MOH}$ is also interested in including the vaccine as part of a comprehensive health package for adolescent health.

\section{Lessons learned from two countries with experience introducing the HPV vaccine Canada - Shainoor Ismail}

Current program: The incidence of cervical cancer in Canada is decreasing. In 2008, most provinces in the country have an HPV vaccine program and they are currently administering Gardasil. They administer the vaccine to girls between ages 9 and 13 (before the beginning of sexual activity) in three doses (0,2 and 6 months) as part of a cervical cancer prevention program. It is offered in schools as a strategy that has proven highly effective in Canada where acceptability is quite high. The vaccine was first introduced with a social marketing campaign that used pamphlets, websites and other dissemination materials. They determined that in order for the project to be costeffective, coverage had to be of at least $70 \%$, a goal that was reached. Researchers have noted the importance of carrying out a project that measures the impact of the intervention, something they anticipate will yield positive results in the long term.

\section{United Kingdom - Loretta Brabin}

Current program: Since September 2008, the United Kingdom has had an HPV immunization program. Cervarix is administered in three doses $(0,1$ and 6 months) to girls between ages 12 and 14 who are attending school. A catch-up program was designed and the vaccine will be offered for three years to adolescents up to 18 years. The vaccine was introduced together with a social marketing campaign stressing the message that it prevents cervical cancer.

Prior challenges: Before the program was introduced, a study was carried out among 3,000 girls aged 12-13 to assess the feasibility and acceptability of the vaccine. Results were quite favorable for both. Initial challenges included opposition from the Catholic Church who opposed the vaccination scheme arguing they were "experimenting" with girls. Another aspect that was debated was the finding that nurses in schools were not always able to respond adequately to parents' concerns especially when dealing with those who opposed the vaccine. All these challenges were successfully addressed and they ended up not having a negative effect on the overall acceptance of the vaccine. Most of the questions put forth by parents had to do with side effects, risks associated with the vaccine and if it would promote sexual promiscuity among the young girls. 
Another challenge included informed consent from the parents: nurses were not comfortable administering the vaccine without parental consent and once parents found out their consent was not needed; this made them more cautious about giving their consent. Maintaining the adolescent's confidentiality was also an important issue. Those parents who initially opposed the vaccine and later gave their consent posed a methodological dilemma since girls who joined the program later were not able to follow the same treatment protocol. The coverage for the first dose was quite high at $83 \%$. As part of the program, women aged 25 and older were reminded to seek screening for cervical cancer. The experience from the United Kingdom illustrates that despite initial concerns, the vaccine was highly acceptable and feasible to implement. Coverage that does not reach traditional immunization programs is still high. However, keeping girls aware and well informed is an ongoing challenge. In sum, the provision of the HPV vaccine constitutes a good starting point to design a more structured program around the sexual and reproductive health needs for adolescents.

\section{Discussion:}

The prior presentations generated a lively discussion about the challenges that lie ahead for countries wanting to implement the HPV vaccine. The following issues could help countries develop their operations research protocol: 1) schools are key sites to offer the vaccine as part of a school health program (for those countries where such a program is in place); 2) countries must reach a consensus on the way in which they plan to implement the social marketing campaign and define the key messages regarding the vaccine they wish to convey. For example, the United Kingdom promoted the program as a way of preventing cervical cancer while other countries may chose to focus on a campaign about sexual and reproductive health and STI prevention among adolescents.

\section{Operations research (OR): proposed methodology}

To inform the countries' development of OR studies to test the HPV vaccine within a comprehensive adolescent health package, Estela Rivero, researcher and professor from El Colegio de México, provided a review of OR addressing basic concepts relevant to design formative research and future interventions (The content of her presentation can be found in Sharepoint, document "Introduction to OR.")

The WHO's proposal for carrying out OR was discussed by Dr. Garrett Mehl (see Sharepoint, document "Design- Adolescent study -HPV"). For the OR project put forth by WHO we need to take into account the three following questions:

- What is the added value of administering the HPV vaccine as part of other specific health interventions for adolescents including sexual and reproductive health services? 
- What are the repercussions of administering the HPV vaccine in an adolescent "plus" package as part of the HPV immunization program (catch up, coverage, etc.)?

- What is the added value of including the HPV vaccine as part of the national program of cervical cancer control?

The group's hypotheses are that offering the "plus" package together with the HPV vaccine will have a positive effect on knowledge and a reduction of sexual risk behaviors among adolescents; that introducing the HPV vaccine together with the "plus" package will not have a negative effect on the use, uptake or coverage of the vaccine in the services offered to school aged children; and, that providing the HPV vaccine will increase the use of screening services for cervical cancer from mothers of the girls that were previously given the HPV vaccine.

The project will have three phases. In phase one, components that will be included in the package will be defined, including the HPV vaccine. During phase 2, OR studies that measure the feasibility, acceptability and determines aspects of monitoring, evaluation and cost of the package will be conducted. Phase 3 will include a more ambitious project that includes a case control methodology. The specific aspects of each stage were described in the power point presentation.

Figura 1. Las tres etapas del proyecto para la introducción de la vacuna contra el VPH y el paquete integrado.

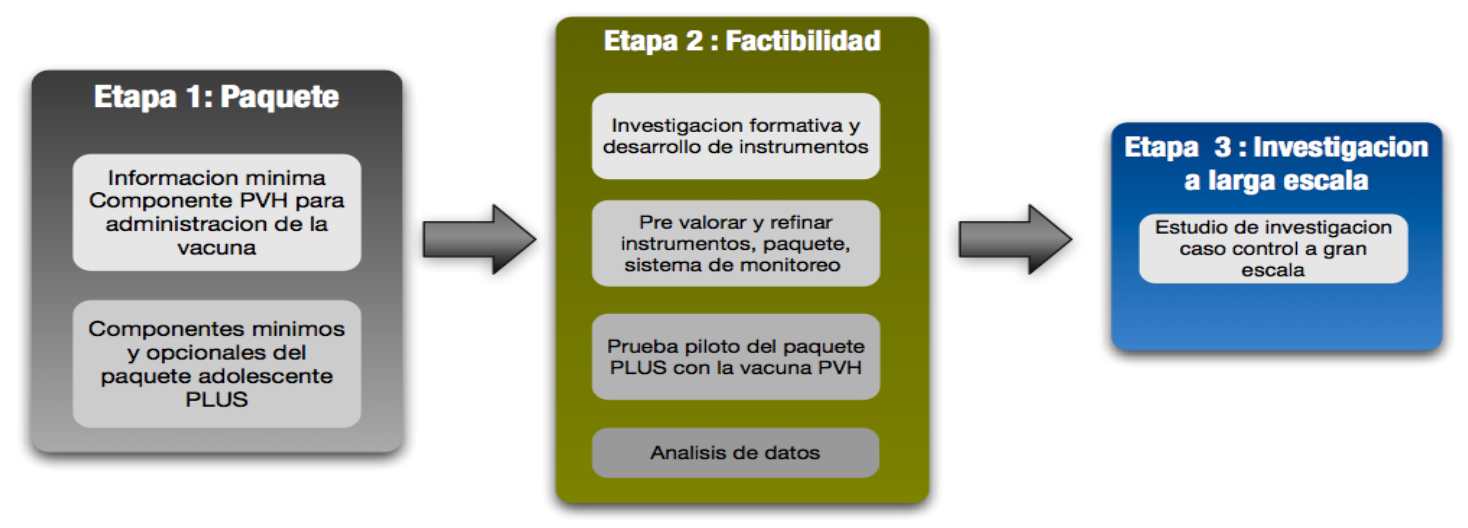

\section{Discussion:}

After the methodological discussion regarding the OR projects, participants posed questions regarding the feasibility of evaluating the impact of the intervention in terms of a measurable behavioral change among the girls that received the HPV vaccine. They concluded that based on some of the components of the package, it would be possible to measure behavioral changes while noting that in the case of sexual and reproductive 
health the observable changes would be in terms of levels of knowledge. Participating countries expressed some doubts regarding the best way of implementing the "plus" package and the ethical issues that arise when budgetary restrictions do not allow them to implement the package to all adolescents. All agreed that the HPV vaccine constitutes a strategic opportunity to improve the coverage and use of health services among adolescents and it is the task of each country to define their local context and implementation mechanisms.

\section{Evaluating the costs of introducing vaccines}

Bárbara Jauregui discussed her experience implementing a PAHO initiative to introduce new vaccines entitled ProVac, aimed at strengthening a country's abilities to make evidence based decisions on whether or not to introduce the HPV vaccine. Her presentation entitled "Assessing costs in an operations research project" discussed the topic of cost-effectiveness in introducing and sustaining new vaccines using the following formula:

\section{$C E A=$ Program costs - costs avoided by preventing disease}

\section{Health benefits (DALYs)}

CEA: Cost effectiveness analysis

DALYs: Disability Adjusted Life Years

For this project, the WHO developed a costing tool kit for health and development services aimed at adolescents. This kit could be used to measure the costs associated with introducing the HPV vaccine alone and in combination with the comprehensive package of health services for adolescents. The kit can be adjusted depending on the package of health services available in each country. The ProVac team is willing to work together with the WHO to sharemethodologies, field work experiences and assess the possibility of integrating tools in the future.

\section{Preliminary ideas for an OR project in the four countries, challenges and next steps}

Using a series of questions as a guide, each country drafted a preliminary plan to explore the potential components that could be included in the comprehensive health package for adolescents in combination with the potential introduction of the HPV vaccine and the initial methodological OR design (see presentations in Sharepoint).

\section{Mexico}

For Mexico, improving its cervical cancer control program thru the HPV vaccine for adolescents is a priority together with an improving cervical cancer diagnosis and treatment among women. The Mexico group concluded that it was indeed possible to carry out a feasibility study to asses the acceptability of the "plus" package that is not currently offered in the new HPV vaccine program. Researchers from Mexico were 
interested in participating with the other countries in this effort. The challenge would be to adapt the package to communities by including the schools, the media and other key players. Their main research questions included, among others: 1) Which interventions from the "plus" package for adolescents and for women could be combined with the HPV vaccine? 2) Are these interventions acceptable among the community and among health professionals? 3) Will health care coverage among adolescents increase with the increased coverage of the HPV vaccine? The group from Mexico would like to carry out a situational analysis to assess the health needs in communities that could serve as a basis for working with local health authorities. While Mexico has an adolescent health package, its coverage is more widespread in urban areas. One of the aims will be to assses the feasibility of adapting the adolescent health package to meet the needs of the most vulnerable communities and those with the lowest human development indices in the country. Components of the health package will include information on SRH, family planning, gender equity, gender based violence, etc. The final components of the package will be determined once the results of the situational analysis are available. The country currenty has a vaccination card for adolescents aged 10 to19 years.

For the case of Mexico the intervention would include: adding the "plus" package of health care services for adolescents to the HPV vaccine that is currently being administered to girls in the poorest 125 communities in the country. More communities with low human development indexes could be added if funding becomes available. The information and services that could be included in the package could be: information on SRH, nutrition, family violence, gender equity, STI and pregnancy prevention. Other vaccines could be also administered together with condoms, folic acid and contraceptives.

The package will be offered by health promoters (promotores de salud). The group mentioned they could join efforts with the cervical cancer program at the $\mathrm{MOH}$ to carry out the study. The key players involved in the study would be: The National Center for the Control and Prevention of HIV/AIDS (Censida), The National Center for the Health of Children and Adolescents, the National Institute of Public Health (INSP), health caravans from the federal MOH, UNICEF, WHO, Population Council and others. This group of experts will design and carry out the pilot phase of the study. In order to offer the "plus" package they will need a multidisciplinary team and other partners such as the National Institute of Epidemiological Reference and IMSS Oportunidades. They will need to hire additional personnel to carry out surveys, do the training, draft data collection instruments and analyze data. Next steps include convening an experts meeting with key players, define more carefully the components of the "plus" package and define the final study methodology.

\section{Discussion:}

Following Mexico's presentation, unfortunately there was no time for group discussion. Nevertheless, the country's team will have to carefully address some of the topics raised during the presentation such as the implications associated with the Mexican MOH decision to use a different schedule than the one used and promoted by WHO. Until this new schedule (two doses followed by a third booster dose at 60 months) is recognized as an internationl standard, the Mexican government and its colleagues will not be able to receive WHO funding for its OR study. The latter could be taken as an opportunity to seek funding from new donors and seek new research partners which can lead to the 
approval of this novel schedule and be considered the evidence based medicine needed to provide the HPV vaccine.

\section{Panama}

Since October 2008, Panama has offered the HPV to girls starting at 10 years enrolled in school. The OR proposed by the country team would use an experimental design by offering the "plus" package in combination with the HPV vaccine. Given that the basic adolescent health package offered in Panama already includes a series of screening tests for vision, hearing, psycho social well being and accident prevention, the "plus" element in the package would be to add the HPV vaccine together with 8 additional sessions on health education that included topics such as cervical cancer, gender and human rights. Panama has several models that could be adapted to the OR methodology. The country team also considered adding recreational and sport activities for girls receiving the vaccine. The study will compare the "plus" package in combination with the HPV vaccine administered to 500 girls vs the "plus" package only administered to 500 girls conforming the control group. Mothers of girls receiving the vaccine will be offered health education sessions that focus on prevention and cervical cancer screening, effective communication strategies with their children, family violene, women's human rights, prevention of gender based violence and sexual exploitation. The OR study will include a 24 week intervention: on day 0 they would administer the HPV vaccine, the first educational session, and a baseline KAP survey. The intervention will also include meetings with parents and violence surveillance. On day 30 they will administer the second dose and the educational sessions with mothers and girls will be given. On day 180 they will carry out the "post" KAP survey to assess change in these indicators. The HPV vaccine with and without the "plus" health package for adolescents will be offered in schools. The Gorgas Institute (a local research institute with a representative attending the meeting) will offer technical assistance calculating the sample size, random selection and implementing the study. The results obtained in the KAP study will be used for purposes of monitoring and evaluation. Researchers will measure attendance at educational sessions and positive change in reported family violence. Keys partners will include: UNFPA, WHO, MOH Panama, Gorgas Institute, among others. Country members expressed their interest in inviting local and international NGOs that have experience working with adolescents such as the Red Cross and the Lions Club. They will convene meetings with these groups to present the OR study methodology and will work on a final version of the study protocol.

\section{Discussion:}

The country team discussed the selection criteria for the girls who will participate in the study. The Gorgas Institute was chosen to calculate the sample. They mentioned they would first have to know the coverage of the current health package for adolescents in Panama targeted for adolescents between 10 to 19, although they believe it will be somewhat difficult to measure real coverage. They concluded that administering the HPV vaccine in schools may help strenghten the demand for other health services among adolescents.

\section{Colombia}


The group from Colombia thought it would be feasible to introduce the HPV vaccine in the country given that the national government supports this health initiative. They also mentioned that some important challenges lay ahead given that no formal agreement has been reached. They propose to start implementing the OR project first. Among the challenges they anticipate they included: political misgivings due to pressure that will be exerted by the pharmaceutical industry; a MOH officials' hesitation regarding purchase of the vaccine; difficulty in accessing girls who live in impoverished or rural areas that are often the ones with the highest rates of HPV; coverage of future cohorts; and, costs of the vaccine. The Colombian governement is in the process of consolidating the vaccination schedule for influenza virus and developing universal access to rotavirus and pneumococus. The latter may delay or hinder the approval of the implementation of the HPV vaccine. Participants also noted that while the above is worth considering, provincial governments do have the autonomy to establish or create additional health priorities provided they follow the guidelines dictated by the federal $\mathrm{MOH}$ and are evidence based medicine interventions (e.g. burden of disese, cost-effectiveness and logistical and financial sustainability).

The research questions posed by the group included: 1) How can we attain adequate coverage of the HPV vaccine among the population most at risk? 2) How can we achieve sustainability given the high cost of the vaccine? 3) How can we improve the existing health services for adolescents maximizing the time the provider spends with the patient? The group wanted to address two working hypothesis: the introduction of the HPV vaccine in combination with the "plus" package will not have a negative effect in the curent vaccine coverage; and, the provision of the "plus" package combined with the HPV vaccine will improve the access and the service utilization among adolescents.

Currently, there is an adolescent health services program that includes visual and anemia screening, growth and development. The "plus" package would add information on SRH, the HPV virus and its association with cervical cancer, and prevention of gender based violence. The convenience sample would consist of adolescent women and the study will be carried out in the province of Medellín in two areas with low levels of human development indices. The study will include an intervention arm ("plus" package) and a control group (current adolescent health services and the HPV vaccine using a social marketing campaign) in each study site. They will offer the two options (both in private and in public clinics) thru their adolescent health service networks. A careful selection of the population will be needed together with insuring the availability of vaccines for future cohorts. The project's success will depend on political will and the possibility of securing funding to purchase the vaccine.

A KAP study will be carried out to measure the effect of the vaccine (including knowledge of SRH and HPV and use of health services among adolescents and their mothers). Other studies will include a cost-effectiveness analysis and an evaluation of the quality of the services being offered. The following institutions will make up the working group: health services in the sites selected to carry out the study; the Ministry of Social Protection; the Colombian National Cancer Institute; and, the WHO and PAHO country offices. They will ask the latter for technical assistance to help with the negotiation with local key players $(\mathrm{MOH})$ and provincial governments. Colombian researchers are interested in sharing expertise and experiences with other countries in the region that have introduced the vaccine and discuss their adolescent health programs. Next steps will include involving the Ministry of Social Protection, design a 
work plan to discuss with the communities that will participate in the study, define budgets and implement the project.

\section{Discussion:}

Participants raised the question about the need to include the community, local NGOs and academic centers once a final methodology is defined. Colombian participants were unaware if the federal government would purchase the vaccine although they thought it would be up to the provincial governments do so. Medellin, is one example of a province who would be willing to purchase it since they already carried out a costeffectiveness study.

Another issue that was raised was: if Colombia has a network of adolescent health services, what would be the added value of implementing the "plus" package or the HPV vaccine? A possibility mentioned was that of raising awareness of the existence of such health services which would in turn increase the demand for services. Participants noted that the network of adolescent health services does not have a wide coverage or a comprehensive approach to prevention, detection and treatment. By introducing the "plus" package and the HPV vaccine, they could improve the quality and coverage of available services. The option of offering the "plus" package in combination with another type of vaccine was mentioned, using Hepatitis B as an example. They did acknowledge however, that by administering a different type of vaccine, they would lose the focus of the project. Researchers mentioned the need to improve current screening services for cervical cancer by implementing the "plus" package and the HPV vaccine.

\section{Peru}

In Peru the HPV vaccine is only administered in private clinics even though the $\mathrm{MOH}$ has a comprehensive health package for adolescents and a national immunization program. The country team mentioned that it would be feasible to assess the introduction of the HPV vaccine and they would be willing to work jointly with other country participants. The challenges that lie ahead include: the country has low coverage and access to current health services for adolescents; and, there seems to be insufficient coordination between the national vaccination program and the life stages of adolescents.

The adolescent health package currently available in Peru shares some similarities with WHO'S “plus” package. They both have screening for hearing and vision, growth charts, psychosocial health and information on SRH. For the study on OR the adolescents who are currently receiving the available health package could serve as the "control" group. Key players involved in the project would include: the $\mathrm{MOH}$ (Immunization Program, SRH Program, Epidemiology Division, offices of communication and provision of drugs, IRB and a group of external consultants. Partners would include, WHO, PAHO, UNICEF, etc. Participants mentioned the need to secure technical assistance and sufficient financial resources to purchase the vaccine.

Next steps would include convening a meeting with the $\mathrm{MOH}$ to discuss the future introduction of the HPV vaccine that would be followed by a social marketing campaign, a cost-effectiveness study, establishment of alliances and parterships and 
technical assistance to distribute the vaccine which would be offered thru the public health sector at the national level. The OR project would focus on three areas where the vaccine would be administered in schools and in health community centers. The research question put forth by participants was: will the "plus" package improve the current health services for adolescents available in Peru? The country team mentioned they would draft a report with preliminary results and conclusions derived from the Mexico City expert's meeting, design a study methodology and meet with key players at the $\mathrm{MOH}$ to define a future strategy. Once this is completed, they would present an OR research protocol to their local IRB and would seek technical assistance from PAHO. The girls who received the HPV vaccine in the year 2008 with the PATH pilot test would conform the "control" group and the girls that will receive the "plus" package would be the intervention group. They will also assess if the mothers of the girls that received the vaccines had their Pap tests.

\section{Discussion:}

Participants from Peru questioned the ethical issue associated with forming a control group since by law, all adolescents are eligible to receive health services. They mentioned it was questionable not to include them in the intervention arm of the study. A few alternatives were discussed, including: design a study where all girls receive the package alone and later in time, receive the "plus" package and the HPV vaccine (once the vaccine is available in the country). Other questions included if the "plus" package had other benefits aside from increasing cervical cancer screening among mothers of girls who receive the vaccine. Another possibility could be that the "plus" package could also increase adherence to other types of vaccines or improve health, lifestyles, and nutrition. The OR study variables should be clarified in order to reach consensus with the $\mathrm{MOH}$ and to overcome the barriers and resistance the vaccine.

\section{Conclusion and next steps}

All country participants expressed their commitment and willingness to move forward with the introduction of the HPV vaccine, the "plus" package, and assess the impact of these interventions through an OR project in their respective countries. However, it is clear that every country has very particular circumstances and is at different stages of introducing the vaccine, which in turn raises other challenges and opportunities. For example, Mexico and Panama already have a strategy in place and are offering the vaccine but will need to consider carefully how best to proceed with the "plus" package. All countries will have to think through the best way they could integrate the HPV vaccine into their current health services for adolescents. Peru and Colombia on the other hand are at the stage of starting discussions with health authorities about the need to introduce the HPV vaccine in their health system as well as securing the essential funding to purchase it. By the end of the meeting, each country had drafted a preliminary OR plan and mapped out next steps. All teams needed to gather additional input and consensus from key stakeholders in their respective countries before elaborating study protocols.

\section{Next steps:}


1. Each country will continue working and refining the implementation of the "plus" package and post it on the meeting Sharepoint. They will share study instruments, experiences and power point presentations; they will use "Elluminate" to facilitate follow-up

2. WHO and PAHO will help countries to further develop the study methodology and design of OR protocol

3. Garrett Mehl will draft a summary of lessons learned in the meeting

4. WHO and PAHO will draft a publication based on the meeting report, including lessons learned and next steps that will be available in Sharepoint and will be open to comments from country participants

5. WHO and PAHO have an international and regional task force that can offer technical assistance and support in specific areas, such as the designing the costeffectiveness study

6. With the aim of serving as a case study, Sharepoint will open a space where countries will be able to upload basic information on sexual and reproductive health indicators, age of menarche, percentage of indigenous girls, prevalence of cervical cancer and HPV vaccine, availability of the HPV vaccine, which will be useful for elaborating their country protocols. 